\title{
Marco Europeo para Organizaciones Educativas Digitalmente Competentes: revisión sistemática 2015-2020
}

\section{European Framework for Digitally Competent Educational Organisations: systematic review 2015-2020}

\author{
(iD) Ángel David Fernández Miravete \\ Universidad de Murcia (España) \\ iD Ma Paz Prendes Espinosa \\ Universidad de Murcia (España)
}

\section{Resumen}

El Marco Europeo para Organizaciones Educativas Digitalmente Competentes (DigCompOrg) es el primer modelo paneuropeo que guía a las instituciones educativas para proceder a su digitalización de una forma estratégica. Esta investigación tiene como objetivo general ofrecer una visión de la producción científica que se basa en este modelo e identificar el enfoque teórico o práctico de estos estudios. Se presenta un estudio exploratorio con una técnica de análisis documental cuyo método consiste en una revisión sistemática de la literatura científica existente publicada en relación con el modelo DigCompOrg desde 2015 hasta 2020. Para la búsqueda se han utilizado las bases de datos bibliométricas Scopus, Web of Science y el motor de búsqueda científica Google Scholar. Tras eliminar duplicidades, se analizan un total de 231 trabajos de los cuales 28 conforman la muestra final tras aplicar los criterios de elegibilidad. Los hallazgos muestran que existe un incremento anual de trabajos que referencian el marco desde el año de su aparición; sin embargo, son pocos los que lo abordan en su planteamiento central. Se destaca de él su versatilidad a la hora de establecer un proceso de autoevaluación sistémico, aunque sería conveniente adaptarlo al contexto educativo real en el que se aplique.

\begin{abstract}
The European Framework for Digitally Competent Educational Organisations (DigCompOrg) is the first pan-European model that guides educational institutions to digitize in a strategic way. The general objective of this research is to offer an overview of the scientific production that is based on this model and to identify the theoretical or practical focus of these studies. An exploratory study with a documentary analysis technique is presented, the method of which consists of a systematic review of the existing scientific literature published in relation to the DigCompOrg model from 2015 to 2020 . For the search, the bibliometric databases Scopus, Web of Science and the scientific search engine Google Scholar were used. After eliminating duplications, a total of 231 works are analyzed, of which 28 make up the final sample after applying the eligibility criteria. The findings show that there is an annual increase in published works that refence the framework from the year of its appearance; however, few of them address it in their central approach. Its versatility in establishing a systemic self-assessment process stands out, although it could be adapted to the real educational context in which it is applied.
\end{abstract}

\section{Palabras clave / Keywords}

Competencia digital, DigCompOrg, evaluación, investigación educativa, organizaciones educativas, liderazgo, revisión sistemática, TIC.

Digital competence, DigCompOrg, evaluation, educational research, educational organizations; leadership, systematic review, ICT. 


\section{Introducción}

La sociedad actual precisa una gran inversión en educación, así como replantearse los sistemas de aprendizaje permanente si pretende dotar al alumnado del siglo XXI de las habilidades necesarias que le permitan una formación de calidad a lo largo de su vida (Comisión Europea, 2020). Entre todas las competencias, la digital es resaltada en multitud de informes elaborados por instituciones y organismos oficiales interesados en el ámbito educativo (Comisión Europea, 2006, 2009, 2018a, 2020; INTEF, 2017; UNESCO, 2011). Es en el año 2010 cuando la Comisión Europea inicia el proyecto DigComp, identificando una serie de descriptores con el objeto de contribuir a la mejor comprensión y desarrollo de la competencia digital entre los ciudadanos europeos. Desde entonces, e independientemente de las distintas versiones publicadas, DigComp se ha utilizado para tres propósitos principales que sintetizan Lucas y Moreida (2017) en: 1) formulación de políticas en el ámbito educativo, la formación y el empleo; 2) el diseño estratégico para las iniciativas de educación, formación y empleo; 3) evaluación y certificación de competencias. A partir de este marco común, se han desarrollado diferentes modelos destacando en el campo de la educación DigCompEdu o «Marco Europeo para la Competencia Digital Docente» (Redecker y Punie, 2017) centrado en la competencia digital del profesorado o DigCompOrg o «Marco Europeo para Organizaciones Educativas Digitalmente Competentes» (Kampylis et al., 2015), traducido al español por el Instituto Nacional de Tecnologías Educativas y de Formación del Profesorado (INTEF) en 2017 dando lugar a una gran cantidad de investigaciones relacionadas con políticas, liderazgo estratégico y prácticas docentes en las aulas (Petterson, 2017).

Si nos centramos en el marco DigCompOrg, por ser objeto del estudio, se presenta un modelo diseñado para ser utilizado por diferentes organizaciones educativas (educación primaria, secundaria y formación profesional o educación superior) que quieran realizar un diagnóstico sobre el uso que hacen de las tecnologías en el proceso de enseñanza y aprendizaje. El concepto de organización digitalmente competente se relaciona con el concepto de "organizaciones de aprendizaje" y "organización basada en competencias" (Stoll y Kools, 2017; Watson, 2014) o con el concepto de "aprendizaje organizacional" (Hong et al., 2017) cuyo objetivo es optimizar el ejercicio de las organizaciones por medio del trabajo colaborativo. En todos estos conceptos subyace la idea de organización digitalmente competente que consigue mejores resultados desde diferentes ángulos. Para ello, DigCompOrg establece siete áreas comunes a todos los sectores de la educación, que incluyen: 1 ) las prácticas de liderazgo y gobernanza; 2) prácticas de enseñanza y aprendizaje; 3) desarrollo profesional; 4) prácticas de evaluación; 5) currículos y contenidos; 6) prácticas de colaboración y comunicación; 7) infraestructura. Además, incluye 15 sub-áreas específicas -y conectadas como partes del mismo conjunto- y 74 descriptores interconectados gráficamente en una rueda (ver Figura 1). El marco es diseñado principalmente para centrarse en el aprendizaje y la enseñanza, así como en las actividades emprendidas por los docentes en un contexto organizacional.

Diferentes informes oficiales europeos, en especial el Plan de Acción de Educación Digital 2018-2020 y el Nuevo Plan de Acción 2021-2027 (Comisión Europea 2017, 2020) afirman que la integración de las tecnologías digitales en la educación requiere un enfoque holístico. Por tanto, una de las acciones clave es promover una planificación y desarrollo de capacidades digitales organizativas actualizadas (Costa, 2021). Al igual que el Marco de Competencias Digitales de la Comisión Europea (DigComp 2.1) (Carretero et al., 2017) reconoce la comprensión del potencial de las tecnologías para promover la inclusión social del individuo, de manera similar, DigCompOrg proporciona una base para la integración a partir de las tecnologías digitales en organizaciones educativas (Kampylis et al., 2015; Mattar et al., 2020). Asimismo, este marco es la base de la herramienta de autoevaluación SELFIE (self-reflection tool \& mentoring scheme for schools) (Comisión Europea, 2018b) que ofrece una visión holística de cómo los estudiantes, docentes y líderes escolares perciben sus políticas y prácticas digitales (Castaño-Muñoz et al., 2021). Así pues, y ante la falta de trabajos de síntesis al respecto, con la presente investigación se pretende identificar y analizar los diferentes estudios teóricos y prácticos publicados que toman como referencia el modelo europeo DigCompOrg para sus investigaciones. 


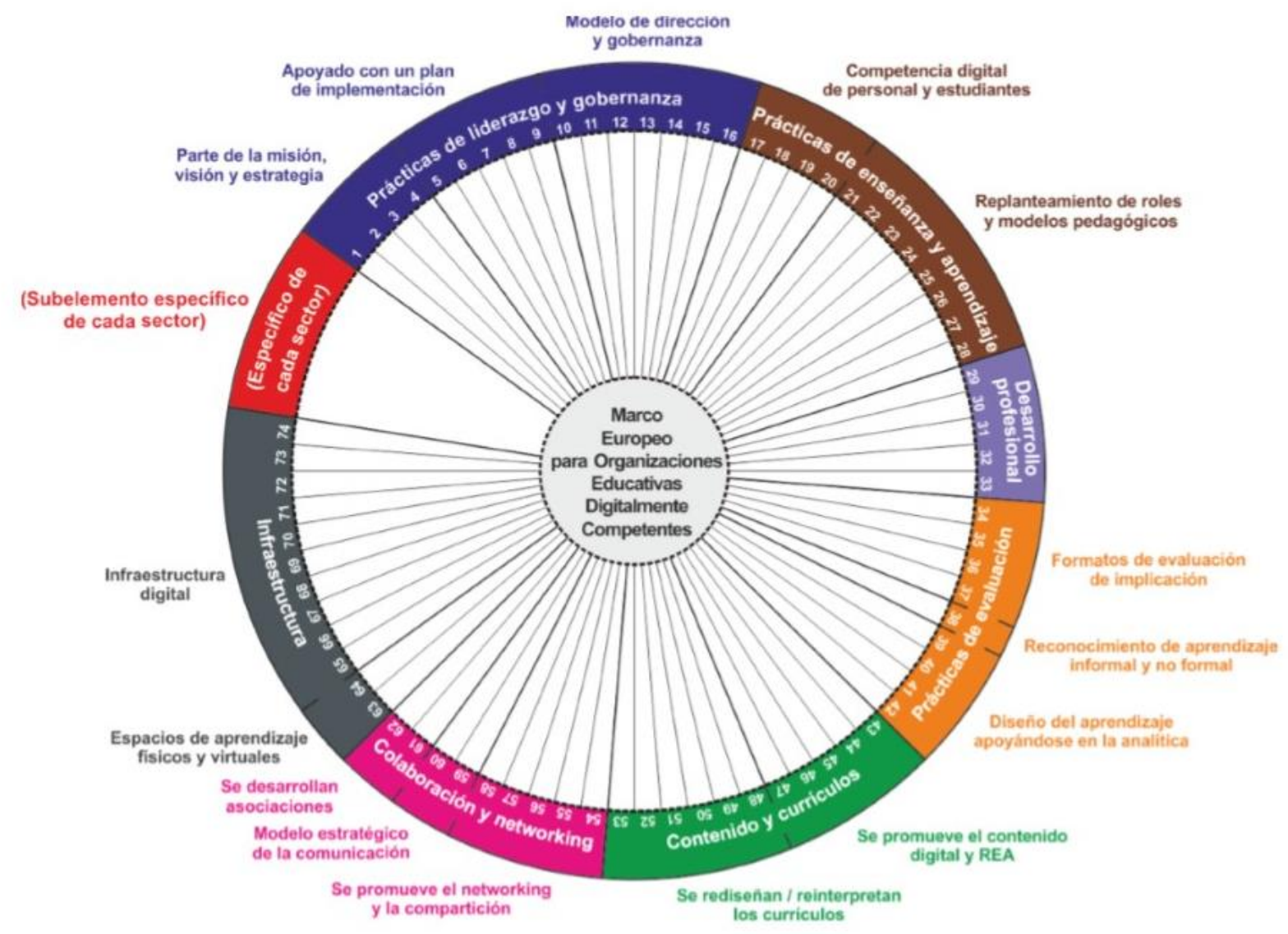

Figura 1. Organizaciones Educativas Digitalmente Competentes: DigCompOrg Fuente: INTEF (2017)

\section{Metodología}

\subsection{Enfoque y objetivos}

Se ha realizado una revisión sistemática, por lo tanto, una investigación básica, apoyada en el protocolo PRISMA (Urrútia y Bonfill, 2010) a partir del siguiente problema, preguntas de investigación y objetivos. El problema de investigación ha sido el análisis del impacto del modelo DigCompOrg en la investigación educativa. Se plantean las siguientes preguntas de investigación: ¿Cuál ha sido el impacto del modelo DigCompOrg en la investigación educativa?, ¿cuántas investigaciones han aplicado el modelo DigCompOrg desde 2015 a 2020?, ¿se ha aplicado desde una perspectiva teórica o práctica o ambas?, ¿qué conclusiones de interés se pueden extraer de la literatura científica sobre DigCompOrg? Para responderlas se marcan los siguientes objetivos concretos:

- Identificar los estudios basados en el modelo DigCompOrg que han sido publicados en el periodo temporal 2015-2020.

- Clasificar los estudios según su enfoque teórico y/o práctico en la aplicación del modelo europeo DigCompOrg.

- Realizar un análisis de contenido para valorar las conclusiones que aportan en relación con la mejora de la realidad de las organizaciones educativas.

Para responder a estas preguntas y objetivos, se ha diseñado una revisión sistemática de la literatura científica existente en la que se analiza la investigación (teórica y empírica) publicada en relación con el modelo DigCompOrg desde 2015 hasta 2020. Este modelo se publicó en 2015, por lo que se justifica iniciar la 
búsqueda en ese año. Se trata de un estudio exploratorio con una técnica de análisis documental (Manchado et al., 2009) cuyo proceso de revisión ha seguido la metodología de Khan et al., (2003) que consiste en 1) enmarcar las preguntas de revisión; 2) identificar la literatura; 3) evaluar los trabajos y su calidad; 4) resumir los estudios revisados 5) interpretar los resultados, lo cual queda reflejado en las siguientes fases.

\subsection{Fases y tareas}

Se ha diseñado el proceso en tres fases, en las que se han llevado a cabo las siguientes tareas:

- Fase 1: Identificación de preguntas y objetivo de investigación. Formulación de criterios de inclusión / exclusión y estrategia de investigación. Extracción de la información especializada procedente de documentos indexados en bases de datos científicas en el periodo 2015-2020.

- Definición de objetivos.

- Definición de criterios de inclusión/exclusión.

- Búsqueda documental e identificación de la literatura.

- Fase 2: Clasificación de la información científica según objetivos de la investigación determinando tendencias.

- Filtrado de información.

- Análisis documental de literatura científica seleccionada.

- Fase 3: Fase final: conclusiones.

- Interpretación de los resultados.

- Discusión y conclusiones.

- Redacción del informe final.

\subsection{Criterios de elegibilidad}

Los criterios iniciales de inclusión fueron: 1) publicaciones relacionadas con el DigCompOrg; 2) publicados en acceso abierto; 3) publicados en cualquier idioma; 4) publicados a partir de 2015 (año de publicación del marco) hasta diciembre de 2020. Los criterios de exclusión son: artículos duplicados o cortos (short papers), informes con diseños poco descriptivos (insuficientes para realizar el análisis de contenido) o de cuyos resultados no pueda establecerse relevancia teórica o práctica para el análisis del impacto del modelo europeo.

\subsection{Fuentes}

El análisis documental ha partido de la selección de literatura realizada a través de búsquedas cruzadas de documentos discriminados de las siguientes fuentes: bases de datos bibliométricas (Scopus y Web of Science) y el motor de búsqueda científica Google Scholar, lo cual ha permitido establecer el análisis de métricas científicas.

\subsection{Búsqueda}

El término seleccionado para la búsqueda fue "DigCompOrg". Es un acrónimo en inglés que permite, por un lado, ampliar el radar de búsqueda a documentos expresados en cualquier idioma y, por otro, restringe los estudios a aquellos que lo referencian. Este término además se puede considerar como un nombre propio, dado que se usa como apelativo del modelo, independiente del idioma en el cual se escriba el documento, tal y como puede observarse en la documentación del portal web de la propia Comisión Europea Europea. ${ }^{1}$

\subsection{Selección de estudios}

Los artículos son seleccionados y evaluados conforme a los criterios de elegibilidad. Tras la búsqueda inicial y la identificación, los trabajos fueron analizados uno a uno. En todos los casos, la frecuencia mínima para su

\footnotetext{
1 https://ec.europa.eu//rc/en/digcomporg
} 
selección inicial fue de, al menos, una aparición del término. Para evitar duplicidades se ha utilizado la herramienta Zotero@ como gestor de referencias bibliográficas. El protocolo para la revisión y criterios para la selección y evaluación de los estudios queda reflejado en la siguiente Tabla 1:

Tabla 1

Protocolo para la revisión y criterios de selección y evaluación de trabajos

\begin{tabular}{|c|c|c|}
\hline Recursos de búsqueda & Bases d & $\begin{array}{l}\text { atos y motores de búsqueda científica } \\
\text { opus, WoS, Google Scholar) }\end{array}$ \\
\hline Descriptor de búsqueda & & DigCompOrg \\
\hline \multirow{5}{*}{$\begin{array}{l}\text { Criterios de inclusión y } \\
\text { exclusión }\end{array}$} & Periodo de tiempo & septiembre de 2015 a diciembre de 2020 \\
\hline & Tipo de documento & $\begin{array}{c}\text { artículos científicos, actas de conferencias, proyectos } \\
\text { educativos }\end{array}$ \\
\hline & Acceso & abierto \\
\hline & $\begin{array}{l}\text { Campo delimitado de } \\
\text { estudio }\end{array}$ & $\begin{array}{c}\text { Marco Europeo para Organizaciones Digitalmente } \\
\text { Competentes (DigCompOrg) }\end{array}$ \\
\hline & Idioma & cualquier idioma \\
\hline
\end{tabular}

Inicialmente, para el rastreo de estudios basados en el modelo DigCompOrg se asignaron conceptos de búsqueda en las bases de datos en los campos de título, resumen y palabras clave y se aplicaron los criterios de inclusión y exclusión. Posteriormente, se procede al análisis de los resúmenes para verificar su relación con nuestro campo de estudio, se identifican las palabras clave y conceptos que reflejan su contribución al tema junto al enfoque de investigación y se identifican sus principales resultados. El flujo del trabajo se refleja en la Figura 2.

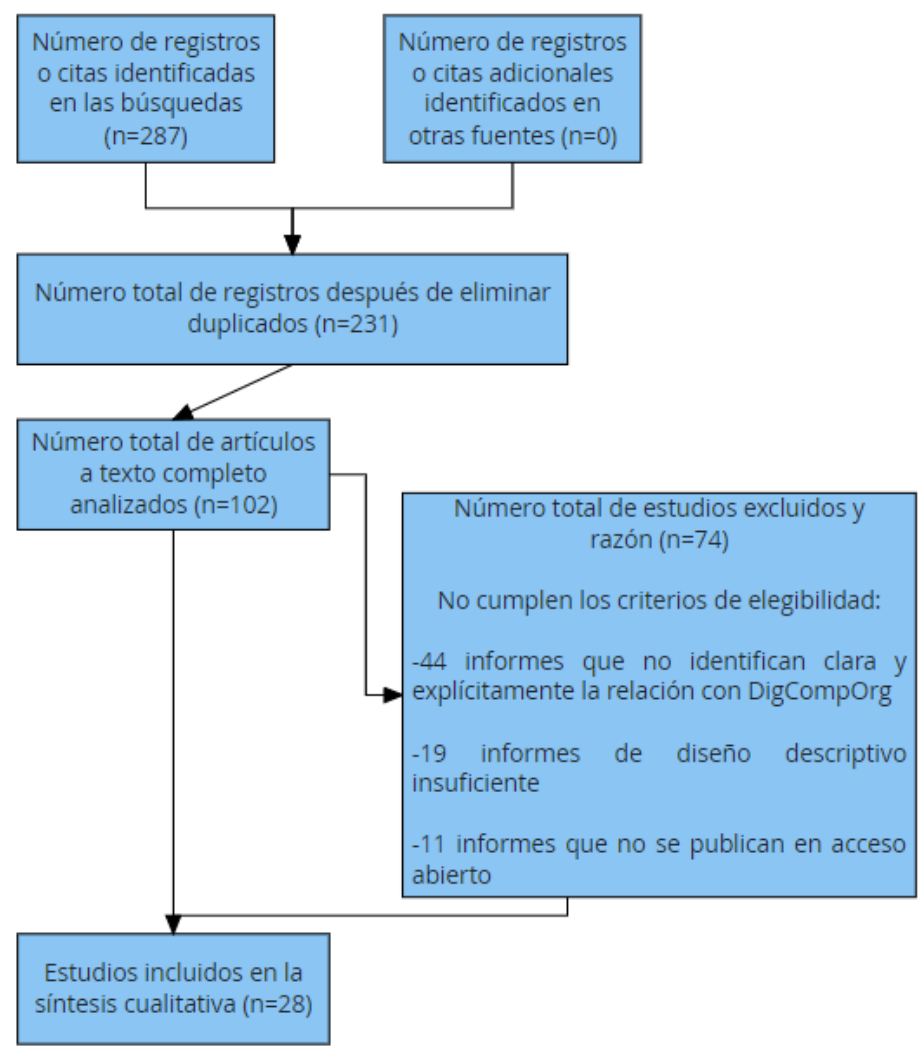

Figura 2. Diagrama de flujo de la revisión sistemática 


\section{Resultados}

La revisión ha permitido identificar un total de 287 documentos (Scopus: 11; Web of Science: 7; Google Scholar: 269). Tras eliminar duplicidades de un mismo resultado, quedan 231 trabajos para analizar. De estos, 129 son excluidos después de revisar el título, resumen y conclusiones por no cumplir los criterios de elegibilidad. Por tanto, son 102 los trabajos para revisar en profundidad. Finalmente, 28 son los trabajos seleccionados que contemplan en su fundamentación teórica el modelo DigCompOrg. Una vez obtenida la muestra final $(n=28)$ se procede a analizar estos trabajos valorando sus resultados y se clasifican atendiendo a una serie de categorías de análisis (ver Tabla 2). Se ha de aclarar que el trabajo encontrado en 2015 es la publicación del propio marco que podemos encontrar traducido a varios idiomas en ese mismo año.

\section{Tabla 2}

Clasificación de los estudios según distintas categorías de análisis

\begin{tabular}{ccccc}
\hline Categoría & Subcategoría & $\begin{array}{c}\text { NNo de } \\
\text { registros o } \\
\text { citas en } \\
\text { búsqueda }\end{array}$ & $\begin{array}{c}\text { No de } \\
\text { registros } \\
\text { sin } \\
\text { duplicar }\end{array}$ & $\begin{array}{c}\text { № de trabajos seleccionados según } \\
\text { criterios de elegibilidad }\end{array}$ \\
\hline Año & 2015 & 5 & 1 & 0 \\
\hline & 2016 & 12 & 11 & 3 \\
\hline \multirow{2}{*}{ Tipo de estudio } & 2017 & 45 & 34 & 5 \\
\hline & 2018 & 61 & 46 & 5 \\
\cline { 2 - 5 } & 2019 & 63 & 57 & 11 \\
\cline { 2 - 5 } & 2020 & 101 & 82 & 14 \\
\hline
\end{tabular}

Según el número de trabajos totales que referencian el descriptor "DigCompOrg", una vez eliminadas las duplicidades y citas, se observa una tendencia alcista de publicaciones desde la aparición del marco europeo en el último trimestre de 2015 (ver Figura 3). Si bien el porcentaje se reduce anualmente hasta el año 2019, vuelve a incrementarse en el año 2020 casi veinte puntos porcentuales $(83.26 \%)$.

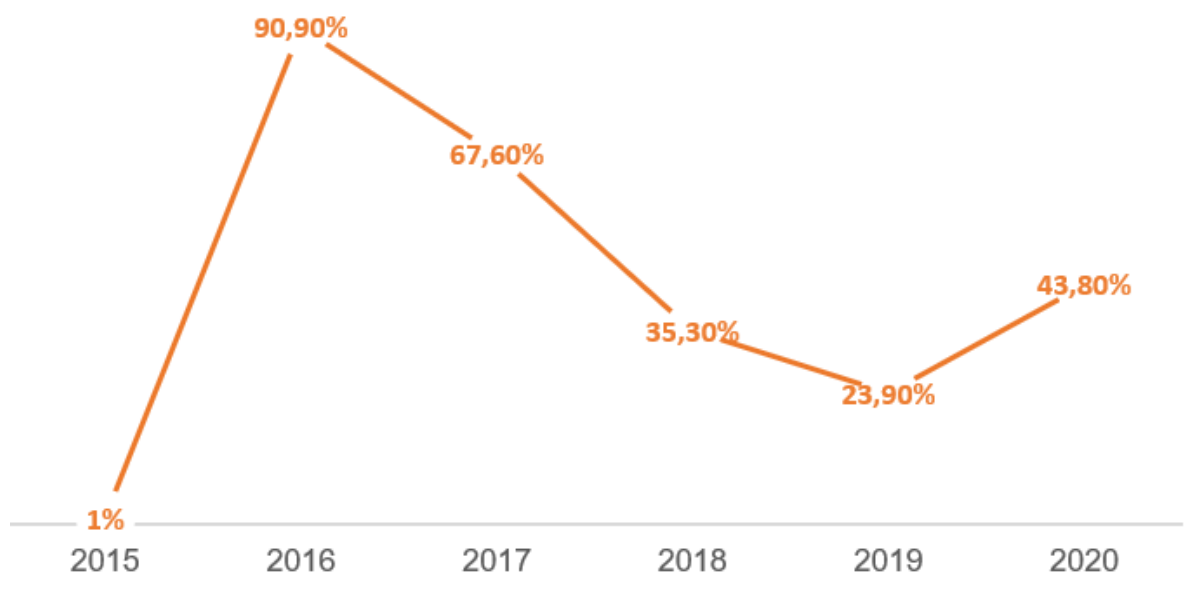

Figura 3. Línea de tendencia de número de publicaciones totales (2015-2020)

Respondiendo a los objetivos de investigación, los trabajos seleccionados se han clasificado en torno a dos categorías: trabajos teóricos y trabajos empíricos basados en experiencias prácticas reales. Cabe señalar que algunos trabajos incorporan contenido teórico o aplicaciones prácticas en su diseño, pero se ha optado en ubicarlos en una u otra categoría en función de su principal propósito. 
Los trabajos teóricos (ver Tabla 3) agrupados aquí toman como referencia el modelo DigCompOrg con diferentes finalidades: analizar planes de implementación TIC (Laici y Orlandini, 2016); identificar áreas específicas de preparación para puestos de trabajo en organizaciones escolares digitalmente competentes (Malach y Kostoloányová, 2017); proponer metodologías de aprendizaje TIC (Racskq y Troll; 2020; SebastiánLópez y De Miguel-González, 2017); reflexionar sobre el papel de la tecnología en el proceso educativo (Fedeli, 2017; Ftáčnik, 2020; Sá y Serpa, 2020); elaborar modelos o herramientas de (auto)evaluación (Čampelj et al., 2019; Ehlers y Bonaudo, 2020; Prendes et al., 2020) o la revisión del mismo marco europeo en comparativa con otros modelos (Đurek et al., 2017; Mattar et al., 2020; Ređep et al., 2019). De sus conclusiones se extrae que DigCompOrg se posiciona como uno de los marcos conceptuales que mejor describen el campo integral de la madurez digital de las escuelas; sin embargo, necesita ser adaptado al contexto local. Por su parte, Serarols (2019) destaca su versatilidad al permitir crear un elemento temático nuevo según el sector educativo en el que se aplique, pero, en cambio, no se establecen niveles de madurez.

Tabla 3

Publicaciones clasificadas como teóricas

\begin{tabular}{|c|c|c|c|c|}
\hline Autor/es & Título & Nivel educativo & Año & País \\
\hline Laici, C. y Orlandini, L. & $\begin{array}{l}\text { "Avanguardie Educative": paths of } \\
\text { innovation for schools }\end{array}$ & $\begin{array}{l}\text { E. Infantil, } \\
\text { Primaria y } \\
\text { Secundaria }\end{array}$ & 2016 & Italia \\
\hline $\begin{array}{l}\text { Đurek, V., Reðep, N. B. y } \\
\text { Divjak, B. }\end{array}$ & $\begin{array}{l}\text { Digital maturity framework for higher } \\
\text { education institutions }\end{array}$ & E. Superior & 2017 & Croacia \\
\hline Fedeli, L. & $\begin{array}{l}\text { School, curriculum and technology: the } \\
\text { what and how of their connections }\end{array}$ & No se especifica & 2017 & Italia \\
\hline $\begin{array}{l}\text { Malach, J. y } \\
\text { Kostoloányová, K. }\end{array}$ & $\begin{array}{l}\text { School as Digitally Competent Educational } \\
\text { Organization: Specific Preparation for Work } \\
\text { Positions and Educational Roles }\end{array}$ & No se especifica & 2017 & $\begin{array}{l}\text { República } \\
\text { Checa }\end{array}$ \\
\hline $\begin{array}{l}\text { Sebastián López, M. y De } \\
\text { Miguel González, R. P. }\end{array}$ & $\begin{array}{l}\text { Educación Geográfica 2020: Iberpix y } \\
\text { Collector for ArcGis como recursos } \\
\text { didácticos para el aprendizaje del espacio }\end{array}$ & E. Secundaria & 2017 & España \\
\hline $\begin{array}{l}\text { Čampelj, B., Karnet, I., } \\
\text { Brodnik, A., Jereb, E. y } \\
\text { Rajkovič, U. }\end{array}$ & $\begin{array}{l}\text { A multi-attribute modelling approach to } \\
\text { evaluate the efficient implementation of ICT } \\
\text { in schools }\end{array}$ & $\begin{array}{l}\text { E. Primaria y } \\
\text { Secundaria }\end{array}$ & 2019 & Eslovenia \\
\hline $\begin{array}{l}\text { Ređep, N. B., Balaban, I., } \\
\text { Žugec, B., Čalopa, M. K. } \\
\text { y Divjak, B. }\end{array}$ & Framework for digitally mature schools & E. Superior & 2019 & Croacia \\
\hline Serarols Boada, J. & $\begin{array}{l}\text { La implementació de les TIC en els centres } \\
\text { d'ensenyament secundari de Catalunya: } \\
\text { propostes d'organització i gestió a partir } \\
\text { d'estudis de cas }\end{array}$ & E. Secundaria & 2019 & España \\
\hline $\begin{array}{l}\text { Ehlers, U. D. y Bonaudo, } \\
\text { P. }\end{array}$ & $\begin{array}{l}\text { DIGI-HE-A Strategic Reflection Tool on } \\
\text { Digitalisation at European Higher Education } \\
\text { Institutions }\end{array}$ & E. Superior & 2020 & Alemania \\
\hline $\begin{array}{l}\text { Ftáčnik, M., Šveda, D. y } \\
\text { Kireš, M. }\end{array}$ & $\begin{array}{l}\text { "Digital transformation of education in } \\
\text { Slovakia within the context of European } \\
\text { documents," } 2020 \text { 18th International } \\
\text { Conference on Emerging eLearning } \\
\text { Technologies and Applications (ICETA) }\end{array}$ & No se especifica & 2020 & Eslovaquia \\
\hline $\begin{array}{l}\text { Mattar, J., Piovezan, M.B, } \\
\text { Souza, S., Santos, C. C. y } \\
\text { dos Santos, A, A. I. }\end{array}$ & $\begin{array}{l}\text { Apresentação crítica do Quadro Europeu de } \\
\text { Competência Digital (DigComp) e modelos } \\
\text { relacionados }\end{array}$ & No se especifica & 2020 & Portugal \\
\hline $\begin{array}{l}\text { Prendes Espinosa, M. P., } \\
\text { Román García, M. y } \\
\text { González Calatayud, V. }\end{array}$ & $\begin{array}{l}\text { Enfoque integrado de análise } \text { da } \\
\text { competência digital em instituições de } \\
\text { ensino superior: professores e alunos no } \\
\text { contexto organizacional }\end{array}$ & E. Superior & 2020 & España \\
\hline Racskq, R. y Troll, E. M. & $\begin{array}{l}\text { Our digital education habits in the light of } \\
\text { their environmental impact: the role of green } \\
\text { computing in education }\end{array}$ & No se especifica & 2020 & Hungría \\
\hline Sá, M. J. y Serpa, S. & $\begin{array}{l}\text { COVID-19 and the Promotion of Digital } \\
\text { Competences in Education }\end{array}$ & E. Superior & 2020 & Portugal \\
\hline
\end{tabular}


Los trabajos empíricos (ver Tabla 4) clasificados en esta categoría incluyen aplicaciones prácticas del marco europeo para realizar intervenciones dirigidas al análisis de experiencias reales concernientes al aprendizaje digital en contextos educativos (Brolpito et al., 2016; Cabero-Almenara et al., 2020; Giunti et al., 2018; Linko et al., 2016); para la construcción de un marco de madurez digital propio con implicaciones prácticas (Balaban et al., 2018; Helenius et al., 2019; Jugo et al., 2017) o aplicación de herramientas de autoevaluación (Chopra, 2019; Trujillo et al., 2020). En todos los estudios se utilizan herramientas que recogen los seis elementos transversales del marco, si bien algunos de ellos (Brolpito et al., 2016; Giunti et al., 2018 y Linko et al., 2016) destacan las áreas de análisis de "prácticas de liderazgo y gobernanza", "prácticas de enseñanza y aprendizaje", "prácticas de evaluación" como las más eficientes a la hora de arrojar datos para una posterior reflexión sobre los planes de integración TIC en el sistema educativo. Finalmente, encontramos estudios que se basan en la aplicación de la herramienta SELFIE (Bocconi et al., 2020; Castaño-Muñoz et al., 2018; Dvoretskaya, 2018; Dvoretskaya y Uvarov, 2020; Panesi et al., 2020). Es de resaltar que todos los estudios seleccionados se contextualizan en países miembros de la Unión Europea a excepción de los de Brolpito et al., 2016; Chopra, 2019; Dvoretskaya, 2018 y Dvoretskaya y Uvarov, 2020 que proceden de Serbia, India y Rusia respectivamente.

\section{Tabla 4}

Publicaciones clasificadas como empíricas

\begin{tabular}{|c|c|c|c|c|}
\hline Autor/es & Título & Nivel educativo & Año & País \\
\hline $\begin{array}{l}\text { Brolpito, A., } \text { Lightfoot, } \\
\text { M., Radišic, J. y } \\
\text { Šcepanovic, D. }\end{array}$ & $\begin{array}{l}\text { Digital and Online Learning in Vocational } \\
\text { Education and Training in Serbia: A Case } \\
\text { Study }\end{array}$ & $\begin{array}{l}\text { Formación } \\
\text { Profesional }\end{array}$ & 2016 & Serbia \\
\hline $\begin{array}{l}\text { Linko, L., Kantola, M. y } \\
\text { Friman, M. }\end{array}$ & $\begin{array}{l}\text { An online journal promoting digital } \\
\text { collaboration in Finnish higher education } \\
\text { institutions }\end{array}$ & E. Superior & 2016 & Finlandia \\
\hline $\begin{array}{l}\text { Jugo, G., Balaban, I., } \\
\text { Pezelj, M. y Redjep, N. B. }\end{array}$ & $\begin{array}{l}\text { Development of a Model to Assess the } \\
\text { Digitally Mature Schools in Croatia }\end{array}$ & $\begin{array}{l}\text { E. Primaria y } \\
\text { Secundaria }\end{array}$ & 2017 & Croacia \\
\hline $\begin{array}{l}\text { Balaban, I., Redjep, N. B. } \\
\text { y Calopa, M. K. }\end{array}$ & $\begin{array}{l}\text { The Analysis of Digital Maturity of Schools in } \\
\text { Croatia }\end{array}$ & $\begin{array}{l}\text { E. Primaria y } \\
\text { Secundaria }\end{array}$ & 2018 & Croacia \\
\hline $\begin{array}{l}\text { Castaño-Muñoz, } \\
\text { Costa, P., Hippe, R. y } \\
\text { Kampylis, P. }\end{array}$ & $\begin{array}{l}\text { Within-school differences in the views on the } \\
\text { use of digital technologies in Europe: } \\
\text { evidence from the SELFIE tool }\end{array}$ & $\begin{array}{l}\text { E. Primaria, } \\
\text { Secundaria y } \\
\text { Formación } \\
\text { Profesional }\end{array}$ & 2018 & $\begin{array}{l}\text { España } \\
\text { (JRC) }\end{array}$ \\
\hline Dovoretskaya, I.V. & $\begin{array}{l}\text { О сформированности общего видения на } \\
\text { использование ИКТ в учебной работе в } \\
\text { школе. (On the formation of shared vision } \\
\text { on the use of information and } \\
\text { communication technologies at school) }\end{array}$ & $\begin{array}{l}\text { E. Primaria, } \\
\text { Secundaria y } \\
\text { Formación } \\
\text { Profesional }\end{array}$ & 2018 & Rusia \\
\hline $\begin{array}{l}\text { Giunti, C., Naldini, M. y } \\
\text { Orlandini, L. }\end{array}$ & $\begin{array}{l}\text { Professional development to support } \\
\text { teaching innovation. The experiences of the } \\
\text { schools leading the Avanguardie Educative } \\
\text { Movement }\end{array}$ & E. Secundaria & 2018 & Italia \\
\hline $\begin{array}{l}\text { Cabero Almenara, J., } \\
\text { Fernández Romero, c. y } \\
\text { Palacio Rodríguez, A. }\end{array}$ & $\begin{array}{l}\text { La competencia digital educativa en } \\
\text { Andalucía (España). El programa\# PRODIG }\end{array}$ & $\begin{array}{l}\text { E. Primaria, } \\
\text { Secundaria, } \\
\text { Formación } \\
\text { Profesional, } \\
\text { Escuela Oficial } \\
\text { de Idiomas }\end{array}$ & 2019 & España \\
\hline Chopra, N. & $\begin{array}{l}\text { E-governance Framework to Measure } \\
\text { Digital Competence of HEls in India }\end{array}$ & E. Superior & 2019 & India \\
\hline $\begin{array}{l}\text { Helenius, K., Linko, L. y } \\
\text { Pirttilä, H. }\end{array}$ & $\begin{array}{l}\text { Viitekehys digikyvykkyyden jäsentämiseksi: } \\
\text { DigCompOrg (Framework for parsing digital } \\
\text { capabilities: DigCompOrg) }\end{array}$ & E. Superior & 2019 & Finlandia \\
\hline $\begin{array}{l}\text { Bocconi, S., Panesi, S. y } \\
\text { Kampylis, P. }\end{array}$ & $\begin{array}{l}\text { Fostering the digital competence of schools: } \\
\text { piloting SELFIE in the Italian education } \\
\text { context }\end{array}$ & $\begin{array}{l}\text { E. Primaria, } \\
\text { Secundaria y } \\
\text { Formación } \\
\text { Profesional }\end{array}$ & 2020 & Italia \\
\hline
\end{tabular}




\begin{tabular}{|c|c|c|c|c|}
\hline $\begin{array}{llll}\text { Dvoretskaya, } & \text { I. } & \text { V. } & \text { y } \\
\text { Uvarov, A. Y. } & & & \end{array}$ & $\begin{array}{l}\text { A combined methodology to assess } \\
\text { innovative ict-supported teaching methods } \\
\text { based on quantitative data }\end{array}$ & $\begin{array}{l}\text { E. Primaria, } \\
\text { Secundaria y } \\
\text { Formación } \\
\text { Profesional }\end{array}$ & 2020 & Rusia \\
\hline $\begin{array}{l}\text { Panesi, S., Bocconi, S. y } \\
\text { Ferlino, L. }\end{array}$ & $\begin{array}{l}\text { Promoting Students' } \\
\text { Inclusion in Schools Through Digital } \\
\text { Technologies: Perceptions of Students, } \\
\text { Teachers, and School Leaders in Italy } \\
\text { Expressed Through SELFIE Piloting } \\
\text { Activities }\end{array}$ & $\begin{array}{l}\text { E. Primaria y } \\
\text { Secundaria }\end{array}$ & 2020 & Italia \\
\hline $\begin{array}{l}\text { Trujillo Sáez, F. J., } \\
\text { Fernández Navas, M., } \\
\text { Montes Rodríguez, R., } \\
\text { Segura Robles, } \\
\text { Alaminos Romero, F. J. y } \\
\text { Postigo Fuentes, A. Y. }\end{array}$ & $\begin{array}{l}\text { Panorama de la educación en España tras } \\
\text { la pandemia de COVID-19: la opinión de la } \\
\text { comunidad educativa }\end{array}$ & $\begin{array}{l}\text { E. Primaria, } \\
\text { Secundaria, } \\
\text { Bachillerato y } \\
\text { Formación } \\
\text { Profesional }\end{array}$ & 2020 & España \\
\hline
\end{tabular}

\section{Discusión y conclusiones}

La aparición de marcos de referencia validados como DigCompOrg contribuye a la clarificación de los principales componentes que constituyen la competencia digital, así como al desarrollo de un lenguaje fiable y compartido sobre la misma. Estos modelos sirven a los profesionales para fines de evaluación (Kullaslahti et al., 2019) y certificación de su propia competencia digital (Prendes et al., 2018); sin embargo, es necesario analizar los trabajos que se están llevando a cabo para conocer el alcance del modelo europeo y producir un conocimiento que permita la transparencia y comparabilidad entre iniciativas relacionadas que estén incorporando este modelo a su propia realidad educativa para avanzar digitalmente como institución. Tal y como se desprende de varios de los estudios analizados (Balaban et al., 2016; Đurek et al., 2017; Jugo et al., 2017; Mattar et al., 2020; Ređep et al., 2019), el concepto de la madurez digital de una escuela se analiza como un indicador que describe el nivel de logro de la integración de las TIC en una organización educativa. Para ello se han de considerar: a) planificación, gestión y liderazgo de las TIC; b) aplicación de las TIC en la enseñanza y el aprendizaje; c) el desarrollo de competencias digitales; d) cultura de las TIC; e) infraestructura TIC. Es por ello que conviene mantener una visión integral de la enseñanza que va más allá de sus principales actores (Brolpito et al., 2016). Sin embargo, si se atiende a los trabajos revisados que referencian a DigCompOrg, si bien el número de publicaciones se incrementa desde su aparición en 2015 (ver Figura 3), son menos los estudios publicados que reflejan claramente su relación con el marco europeo. Esto puede indicar, por un lado, que todavía existe mayor preocupación por el estudio del desarrollo de la competencia digital docente que por investigar este proceso dentro del sistema organizacional en el que tiene lugar y, por otro, que la aplicación de modelos de madurez digital es una línea de investigación todavía en desarrollo.

Tras el análisis de resultados se observa cierto equilibrio entre publicaciones que presentan trabajos que desarrollan experiencias prácticas de mejora educativa y desarrollos teóricos fundamentados en el modelo europeo. En cuanto a los estudios teóricos se pueden clasificar entre aquellos que analizan el marco europeo junto con otros marcos con la finalidad de construir uno propio (Ftáčnik et al., 2020; Jugo et al., 2017; Serarols Boada, 2019); revisar currículos o metodologías (Fedeli, 2017; Helenius et al., 2019; Sá y Serpa, 2020; Sebastián-López y De Miguel-González, 2017); creación de modelos y herramientas evaluativas (Čampelj, 2019; Ehlers y Bonaudo, 2020; Prendes et al., 2020) o reflexionar sobre idearios educativos (Laici y Orlandini, 2016; Racskq y Troll, 2020). Los clasificados como estudios empíricos abordan diferentes experiencias que sirven para elaborar planes educativos de mejora incluyendo las TIC como eje estratégico que abarcan educación primaria, secundaria, formación profesional y educación superior (Balaban et al., 2018; Brolpito, 2016; Chopra, 2019; Dovoretskaya, 2019; Giunti et al., 2018; Jugo et al., 2017; Prendes et al., 2020; Ređep et al., 2019). Además, aquí se incluyen aquellos trabajos de implicación práctica que utilizan la herramienta SELFIE y de la que destacan su versatilidad a la hora de establecer una autoevaluación estructural (Bocconi et al., 2020; Castaño-Muñoz et al., 2018; Dvoretskaya, 2018; Dvoretskaya y Uvarov, 2020; Panesi et al., 2020). De los estudios revisados, hay que señalar que casi la totalidad provienen del continente europeo, pese a que varios reflejan que se trata de un marco aplicable en cualquier contexto (Durek et al., 2017; Helenius et al., 2019; Linko et al., 2016).

En cuanto a las áreas de reflexión en las que se divide el marco, diversos autores resaltan cuatro como las de mayor valor práctico (Brolpito et al., 2018; Chopra, 2019; Giuti et al., 2018; Jugo et al., 2017; Linko et al., 
2016): 1) las prácticas de liderazgo y gobernanza; 2) prácticas de enseñanza y aprendizaje; 3) prácticas de evaluación y 4) prácticas de colaboración y comunicación. Esto pone de manfiefiesto que el modelo DigCompOrg destaca la importancia no solo de los actores principales en el proceso educativo (profesorado y alumnado), sino que también pone el acento en el equipo de gestión (líderes educativos), la evaluación del proceso y la necesidad de crear oportunidades de capacitación, intercambio de conocimiento, discusión y desarrollo de prácticas con tecnología digital. En este último sentido, se reconoce la importancia que tiene la escuela como responsable de crear redes sociales, colaboraciones y contactos con su entorno local. En todos los casos, los informes analizados reconocen el valor del marco en sus múltiples vías para innovar orgánicamente a lo largo del tiempo enfatizando, por un lado, que no existe una ruta única o mejor para la innovación y, por otro lado, que el cambio es sistémico o no es un cambio (Dvoretskaya, 2018).

Este trabajo ha presentado datos que pueden servir de base para futuros estudios sobre el marco europeo DigCompOrg. Además, la base de datos puede servir de soporte para otras investigaciones interesadas en analizar otros aspectos teóricos y/o prácticos (tipos de experiencias, instrumentos, metodología, etc.).. Sin embargo, la incorporación de otros procedimientos estadísticos para el tratamiento de datos o la realización de un meta-análisis hubiera permitido establecer conclusiones de mayor profundidad. Este hecho puede constituir una limitación; no obstante, los resultados que se presentan demuestran que los estudios sobre competencia digital en relación a las infraestructuras organizacionales y el liderazgo estratégico son todavía reducidos, lo que manifiesta una línea de investigación novedosa. Para finalizar se concluye que el logro de la competencia digital no debería considerarse como una acción aislada que tiene lugar en un nivel de actores individuales. Por el contrario, se puede considerar como un proceso en desarrollo que está impulsado e influido por diversos factores contextuales que se encuentran en y más allá de una organización educativa cuyos integrantes debieran compartir objetivos, metas y cultura en un proceso de enseñanza y aprendizaje transversal y sistémico.

\section{Referencias}

Balaban, I., Redjep, N. B., y Calopa, M. K. (2018). The analysis of digital maturity of schools in Croatia. International Journal of Emerging Technologies in Learning, 13(6), 4-15. https://doi.org/10.3991/ijet.v13i06.7844

Bocconi, S., Panesi, S., y Kampylis, P. (2020). Fostering the digital competence of schools: piloting SELFIE in the Italian education context. IEEE Revista Iberoamericana de Tecnologias del Aprendizaje, 15(4), 417-425. https://doi.org/10.1109/RITA.2020.3033228

Brolpito, A., Lightfoot, M., Radišic, J., y Šcepanovic, D. (2016). Digital and Online Learning in Vocational Education and Training in Serbia: A Case Study. European Training Foundation. Retrieved from https://bit.ly/36Y20Se

Cabero-Almenara, J., Fernández Romero, C. y Palacios Rodriguez, A. D. P. (2020). La competencia digital educativa en Andalucía (España). El programa\# PRODIG. Temas de Comunicación, (41). Retrieved from https://bit.ly/2V7b44w

Čampelj, B., Karnet, I., Brodnik, A., Jereb, E., y Rajkovič, U. (2019), A multi-attribute modelling approach to evaluate the efficient implementation of ICT in schools. Central European Journal of Operations Research, 27(3), 851-862. https://doi.org/10.1080/15700763.2015.1024328

Carretero, S., Vuorikari, R., y Punie, Y. (2017). DigComp 2.1. The digital Competence Framework for Citizens. With eight proficiency levels and examples of use. Publications Office of the European Union, EUR 28558 EN. https://doi.org/10.2760/38842

Castaño-Muñoz, J., Weikert García, L., y Herrero Rámila, C. (2021). Analysing the digital capacity of Spanish schools using SELFIE. EUR 30735 EN, Publications Office of the European Union, Luxembourg, 2021, ISBN 978-92-7638651-3 (online). https://doi.org/10.2760/947402

Chopra, N. (2019). E-governance Framework to Measure Digital Competence of HEls in India. European Scientific Journal, 15(11), 181-193. Retrieved from http://dx.doi.org/10.19044/esj.2019.v15n11p181

Comisión Europea (2020). Plan de Acción de Educación Digital (2021-2027). Unión Europea. Retrieved from https://bit.ly/2VceC5H

Comisión Europea (2018a). Recomendación relativa a las competencias clave para el aprendizaje permanente. Diario Oficial de la Unión Europea, 4(6). Retrieved from https://bit.ly/3ia76RQ

Comisión Europea (2018b). SELFIE, Self-reflection on Effective Learning by Fostering the use of Innovative Educational Technologies. Bruselas, Parlamento Europeo. Retrieved from https://bit.ly/3x9gN7o

Comisión Europea (2017). Una agenda digital para Europa. Bruselas, Parlamento Europeo. Retrieved from https://bit.ly/3i8nxho

Comisión Europea (2009). Marco estratégico para la cooperación de la Unión Europea en el ámbito de la educación y la formación (ET2020). Diario oficial de la Unión Europea, (2009/C 119/02). Retrieved from https://bit.ly/315c58u

Comisión Europea (2006). Competencias clave para el aprendizaje permanente. Recomendación 2006/962/CE del Parlamento Europeo y del Consejo, de 18 de diciembre de 2006, sobre las competencias clave para el aprendizaje permanente. Diario Oficial de la Unión Europea (L 394 de 30.12.2006). Retrieved from https://bit.ly/314OTqE 
Costa, P., Castaño-Muñoz, J., y Kampylis, P. (2021). Capturing schools' digital capacity: Psychometric analyses of the SELFIE self-reflection tool. Computers \& Education, 162, 104080. https://doi.org/10.1016/j.compedu.2020.104080

Doretskaya, I. V. (2018). On the formation of a common vision on the use of ICT in academic work at school. Science and school, (5), 127-132. Retrieved from https://bit.ly/2TFsWTX

Dvoretskaya, I. V., y Uvarov, A. Y. (2020). A combined methodology to assess innovative ict-supported teaching methods based on quantitative data. МатериалыIV Международной научнойконфреренции Красноярск, 69октября2020z., 553. Retrieved from https://bit.ly/3iYSgg6

Đurek, V., Reðep, N. B., y Divjak, B. (2017). Digital maturity framework for higher education institutions. Central European Conference on Information and Intelligent Systems. Faculty of Organization and Informatics Varazdin, 99-106. Retrieved from https://bit.ly/3zMroXp

Ehlers, U. D., y Bonaudo, P. (2020, June). DIGI-HE-A Strategic Reflection Tool on Digitalisation at European Higher Education Institutions. En EDEN Conference Proceedings (No. 1, pp. 289-298). https://doi.org/10.38069/edenconf2020-ac0027

Fedeli, L. (2017). School, curriculum and technology: the what and how of their connections. Education Sciences \& SocietyOpen Access Journal, 8(2). Retrieved from https://bit.ly/3iYU8p8

Ftáčnik, M., Šveda, D., y Kireš, M. (2020). Digital transformation of education in Slovakia within the context of European documents. En 2020 18th International Conference on Emerging eLearning Technologies and Applications (ICETA) (pp. 113-118). IEEE. https://doi.org/10.1109/ICETA51985.2020.9379154

Giunti, Ch., Naldini, M., y Orlandini, L. (2018). Professional development to support teaching innovation. The experiences of the schools leading the Avanguardie Educative Movement. Form@ re-Open Journal per la formazione in rete, 18(2), 103-115. Retrieved from http://dx.doi.org/10.13128/formare-23109

Helenius, K., Linko, L., y Pirttilä, H. (2019). Viitekehys digikyvykkyyden jäsentämiseksi: DigCompOrg. AMK-lehti / UAS Journal. Retrieved from https://bit.ly/2Wyl1ch

Hong, J., Snell, R., y Rowley, C. (2017). Organizational learning in Asia. Atlanta, Elsevier.

Instituto Nacional de Tecnologías Educativas y de Formación del Profesorado (INTEF) (2017). Marco Común de Competencia Digital Docente. Retrieved from https://bit.ly/2WuNuj5

Jugo, G., Balaban, I., Pezelj, M., y Redjep, N. B. (2017, July). Development of a Model to Assess the Digitally Mature Schools in Croatia. IFIP World Conference on Computers in Education, 169-178. https://doi.org/10.1007/978-3-31974310-3 19

Kampylis, P., Punie, Y., Devine, J. (2015). Promoción de un aprendizaje eficaz en la era digital. Un marco europeo para organizaciones educativas digitalmente competentes, vol. 27599, Ministerio de Educación. Retrieved from https://bit.ly/2UQDGj0 Traducido por INTEF (2016). https://doi.org/10.4438/030-16-426-9

Khan, K. S., Kunz, R., Kleijnen, J., y Antes, G. (2003). Five steps to conducting a systematic review. Journal of the Royal Society of Medicine, 96(3), 118-121. https://doi.org/10.1258/jrsm.96.3.118

Kullaslahti, J., Ruhalahti, S., y Brauer, S. (2019). Professional Development of Digital Competences: Standardised Frameworks Supporting Evolving Digital Badging Practices. Humanities \& Social Sciences, 12(2), 175-186. Retrieved from https://bit.ly/3ya5KMH

Laici, C., y Orlandini, L. (2016). Avanguardie Educative: paths of innovation for schools. Research on Education and Media, 8(1), 53-61. https://doi.org/10.1515/rem-2016-0007

Linko, L., Kantola, M., y Friman, M. (2016). An online journal promoting digital collaboration in Finnish higher education institutions. EAPRIL 2016, 228-238. Retrieved from https://bit.ly/2WtMDz7

Lucas, M., y Moreida, A. A. (2017). Information and Communication Overload: Can DigComp Help? En R. P. Figueiredo Marques y J. C. Lopes Batista (Eds.), Information and Communication Overload in the Digital Age (157-175). IGI Global Editorial. https://doi.org/10.4018/978-1-5225-2061-0.ch007

Manchado Garabito, R., Tamames Gómez, S., López González, M., Mohedano Macías, L., D’Agostino, M., y Veiga de Cabo, J. (2009). Revisiones sistemáticas exploratorias. Medicina y seguridad del trabajo, 55(216), 12-19. Retrieved from https://bit.ly/3BOAO6B

Malach, J., y Kostoloányová, K. (2017). School as Digitally Competent Educational Organization: Specific Preparation for Work Positions and Educational Roles. European Conference on e-Learning, Academic Conferences, International Limited, 344-352. Retrieved from https://bit.ly/3/4oZDF

Mattar, J., Piovezan, M. B., Souza, S., Santos, C. C., y dos Santos, A. I. (2020). Apresentação crítica do Quadro Europeu de Competência Digital (DigComp) e modelos relacionados. Research, Society and Development, 9(4), e172943062e172943062. Retrieved from http://dx.doi.org/10.33448/rsd-v9i4.3062

Panesi, S., Bocconi, S., y Ferlino, L. (2020). Promoting Students' Well-Being and Inclusion in Schools Through Digital Technologies: Perceptions of Students, Teachers, and School Leaders in Italy Expressed Through SELFIE Piloting Activities. Frontiers in Psychology, 11. https://doi.org/10.3389/fpsyg.2020.01563

Petterson, F. (2017). On the issues of digital competence in educational contexts: a review of literature. Education and information technologies, 23(3), 1005-1021. https://doi.org/10.1007/s10639-017-9649-3

Prendes Espinosa, M. P., García Román, M. y González Calatayud (2020). Enfoque integrado de análise da competência digital em instituições de ensino superior: professores e alunos no contexto organizacional. Revista PanorâmicaISSN, 32(12), 2238-9210. Retrieved from https://bit.ly/3f6T8OH 
Prendes Espinosa, M. P., Gutiérrez Porlán, I. y Martínez Sánchez, F. (2018). Competencia digital: una necesidad del profesorado universitario del siglo XXI. RED, Revista de Educación a Distancia, (56), 1-22. Retrieved from http://dx.doi.org/10.6018/red/56/7

Racskq, R., y Troll, E. M. (2020). Our digital education habits in the light of their environmental impact: the role of green computing in education. Teaching Mathematics and Computer Science, 18(1), 69-86. Retrieved from https://bit.ly/3iamLk7

Redecker, C., y Punie, Y. (2017). Digital competence framework for educators (DigCompEdu). Seville: RC-IPTS. Retrieved from https://bit.ly/3xlY5tv

Ređep, N. B., Balaban, I., Žugec, B., Čalopa, M. K., y Divjak, B. (2019). Framework for digitally mature schools. European Journal of Open, Distance and E-learning, (1), 360-371. Retrieved from https://bit.ly/3BRaBoa

Sá, M. J., y Serpa, S. (2020). COVID-19 and the Promotion of Digital Competences in Education. Universal Journal of Educational Research, 8(10), 4520-4528. https://doi.org/10.13189/ujer.2020.081020

Sebastián-López, S., y de Miguel-González, R. (2017). Educación Geográfica 2020: Iberpix y Collector for ArcGis como recursos didácticos para el aprendizaje del espacio. Didáctica Geográfica (18), 231-246. Retrieved from https://bit.ly/3yiqemw

Serarols Boada, J. (2019). La implementació de les TIC en els centres d'ensenyament secundari de Catalunya: propostes d'organització i gestió a partir d'estudis de cas. Tesis de Doctorado, Barcelona, Universitat Oberta de Catalunya (UOC).

Stoll, L., y Kools, M. (2017). The school as a learning organisation: a review revisiting and extending a timely concept. Journal of Professional Capital and Community, 2(1), 2-17. https://doi.org/10.1108/JPCC-09-2016-0022

Trujillo Sáez, F. J., Fernández Navas, M., Montes Rodríguez, R., Segura Robles, A., Alaminos Romero, F. J., y Postigo Fuentes, A. Y. (2020). Panorama de la educación en España tras la pandemia de COVID-19: la opinión de la comunidad educativa. Madrid: Fad. https://doi.org/10.5281/zenodo-3878844

United Nations Educational, Scientific and Cultural Organization (UNESCO) (2011). UNESCO ICT Competency Framework for Teachers. UNESCO Institute for Information Technologies in Education. Retrieved from https://bit.ly/3x9sCKU

Urrútia, G., y Bonfill, X. (2010). Declaración PRISMA: una propuesta para mejorar la publicación de revisiones sistemáticas y metaanálisis. Medicina Clínica, 135(11), 507-511. https://doi.org/10.1016/j.medcli.2010.01.015

Watson, C. (2014). Effective professional learning communities? The possibilities for teachers as agents of change in schools. British Educational Research Journal, 40(1), 18-29. https://doi.org/10.1002/berj.3025 